\title{
Online verification of multiple safety criteria for a robot trajectory
}

\author{
Dario Beckert, Aaron Pereira and Matthias Althoff
}

\begin{abstract}
Ensuring the safety of humans in a collaborative environment with industrial robots is a major concern of human-robot co-working. Most current approaches give no formal guarantee of safety; where such guarantees are given, accounting for the unpredictability of the human may limit robot efficiency. We therefore developed a novel trajectory planner for a robot arm, which formally guarantees the safety of humans from collision with the robot for every possible human behaviour, without restricting the robot more than necessary. To achieve this, the trajectory planner verifies online that the robot adheres to two separate safety criteria derived from ISO standards using reachable occupancies of surrounding humans and the robot. While one criterion anticipates all possible movements of the human and provides a reasonable safety guarantee, the other criterion additionally guarantees strict safety as long as the human behaves as expected according to ISO standards. We implemented the trajectory planner for a real robot arm and show some experimental results.
\end{abstract}

\section{INTRODUCTION}

A limiting factor for the introduction of robots working alongside humans in industry is the challenge of guaranteeing safety. To avoid injury from collisions, robots must react to the behaviour of nearby humans, whose future motion is hard to predict. Accounting for only the most likely subset of the motion, as in most previous approaches, introduces the risk that the robot does not behave safely during unexpected motion, whereas accounting for all physically possible motion could restrict the robot performance to a large extent.

We present a novel approach whereby we simultaneously guarantee a strong safety condition accounting for expected human motion, and a weaker safety condition accounting for all possible movements. This avoids over-cautious robot behaviour which would lead to poor performance, while nevertheless guaranteeing safety for nearby humans. We guarantee these conditions in our formally verified trajectory planner for an industrial robot arm. The trajectory planner is based on the principle that no movement is executed without previously being formally verified to be safe.

Since the human moves fast and with uncertain intention, most approaches which predict future human movement to avoid collisions are probabilistic. Ding et al. present a Hidden Markov Model [1] to predict hand occupancy in reaching motions. Mainprice and Berenson use a Gaussian Mixture Model to estimate the probability of space around the robot being occupied by a human [2]; this is used in a trajectory planner. On a higher level, [3] uses information from objects in the environment to predict most likely human motion.

The authors are with the Department of Informatics, Technical University of Munich, 85748 Garching, Germany. d.beckert@tum. de, aaron.pereiraltum.de, althoffein.tum.de
Though probabilistic approaches often work well, they cannot be relied upon to provide a strict guarantee of safety, e.g. when a human performs an unexpected movement.

To account for this, Petti and Fraichard [4] propose planning short-term plans where an agent can provably avoid states which would lead to collision (Inevitable Collision States). This principle has been applied to autonomous vehicles [5] and robot manipulators [6]. In most cases, a robot has been considered safe when it is stationary. However, for close co-existence, this requirement may be too restrictive-when the human is near the robot, accounting for all movements may mean that the robot cannot move at all.

If collisions cannot be avoided, then collision severity should be minimised. Soft robots [7] are designed to be inherently safe, but the difficulty of controlling them accurately limits application. Impact energy minimisation is considered in [8], and the recent ISO Technical Standard [9] describes limiting of impact force and pressure, among other safety criteria. The ISO standard governing humanrobot collaborative operation [10] describes a Safety-rated Reduced Speed mode of operation which can be used where humans are nearby, with the justification that this should "allow sufficient time for people either to withdraw from the hazardous area or to stop the robot". Here, the Cartesian speed of the tool centre point (TCP) is reduced to below $0.25 \frac{\mathrm{m}}{\mathrm{s}}$.

Our novel approach, however, guarantees that the robot will stop before the human can reach it, when the human behaves according to expectations. In the case that the human executes unexpected movement, it guarantees that the Cartesian speed of the robot at the point of impact is less than a certain value. Next, we introduce the trajectory planner and give a formal definition of the safety criteria. In Sec. III we present the developed trajectory planning algorithm step by step; in Sec. IV we show how to calculate robot and human occupancies in a conservative way. In Section V we describe our experimental results and conclude in Sec. VI.

\section{PROBLEM STATEMENT AND TERMINOLOGY}

We develop a robot trajectory planner that is able to adapt online to human behaviour to meet two safety criteria. To accomplish this, we verify the trajectory piecewise. A longterm trajectory is given by a higher-level planner. Since it would unnecessarily restrict the robot's movement if we were to certify the safety of the entire long-term trajectory, we calculate short-term plans in smaller steps ahead and formally verify the safety conditions for each of those, as shown in Fig. 1. 


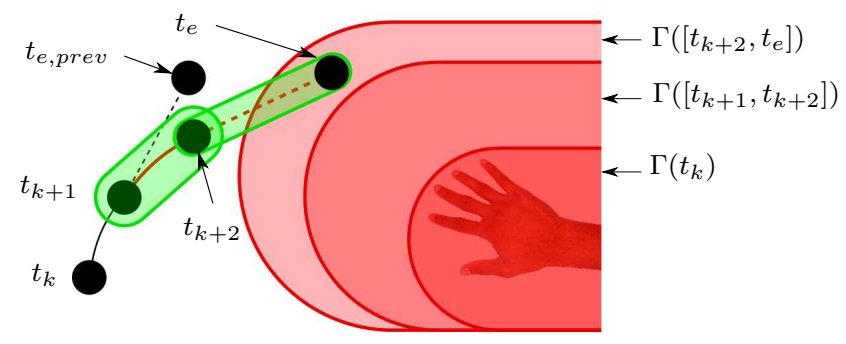

Fig. 1. The robot is currently moving on $\left[t_{k}, t_{k+1}\right]$ while verifying the short-term plan $\left[t_{k+1}, t_{e}\right]$. Since the reachable occupancies of robot (green capsules) and human (red capsules) intersect, this movement cannot be guaranteed to be safe and the robot executes the previously verified failsafe manoeuvre $\left[t_{k+1}, t_{e, p r e v}\right]$. The failsafe manoeuvre is shown off-path for illustration purposes; it is actually path-consistent with the desired trajectory.

A short-term plan consists of a piece of the desired longterm plan followed by a failsafe manoeuvre that brings the robot to a stop. As proposed in [4], we verify the subsequent short-term plan while executing the current one. Prior to $t_{k}$, we have verified that the short term plan from $t_{k}$ to $t_{e, p r e v}$ is safe. While executing the first part from $t_{k}$ to $t_{k+1}$, we verify the next short-term plan, which consists of the piece of the long-term plan from $t_{k+1}$ until $t_{k+2}$, followed by a failsafe manoeuvre from $t_{k+2}$ to $t_{e}$. If the verification fails, at $t_{k+1}$ the robot executes the failsafe manoeuvre from $t_{k+1}$ to $t_{e, p r e v}$, otherwise it moves along the now guaranteed safe piece of the desired plan in $\left[t_{k+1}, t_{k+2}\right]$. We describe in III-A how these short-term plans are generated.

As previously mentioned, we verify two criteria: that the robot stops before the human reaches it, as long as the human moves as expected, and that the robot velocity is under a predefined speed, in case the human moves unexpectedly. We take expected movement to be defined by the maximum speed of the upper body given in ISO 13855 [11]. In [12], we show that this criterion is less conservative than the actual extreme movements of the human. To account for unexpected movement, we use a model from [13], which accounts for all human movements found in human-robot co-existence scenarios. To be able to provide a formal definition of safety we first define reachable occupancies:

Definition 1: ISO OCCUPANCY AND REACHABLE OCCUPANCY: Consider the human body as a dynamical system with an unknown, internal state $\mathbf{w}(t)$, and dynamics $\dot{\mathbf{w}}=$ $g(\mathbf{w}, \boldsymbol{u})$ where $\boldsymbol{u}(t)$ is some input in the set $\mathcal{U}$ of all possible inputs. Let $\Xi_{0}$ be the initial set of possible body states $\mathbf{w}\left(t_{0}\right)$ given readings from the sensors at time $t_{0}$, allowing for measurement uncertainty. Let $\mathcal{F}(\mathbf{w}) \subset \mathbb{R}^{3}$ be the spatial occupancy of the human at a particular state. The system's reachable set is: $W\left(\left[t_{a}, t_{b}\right]\right)=\left\{\mathbf{w}\left(t_{0}\right)+\right.$ $\left.\int_{t_{0}}^{t} g(\mathbf{w}, \boldsymbol{u}) d t \mid \mathbf{w}\left(t_{0}\right) \in \Xi_{0}, t \in\left[t_{a}, t_{b}\right], \boldsymbol{u}(t) \in \mathcal{U}\right\}$. We define the reachable occupancy as:

$$
\Gamma_{r}\left(\left[t_{a}, t_{b}\right]\right) \supseteq\left\{\mathcal{F}(\mathbf{w}) \mid \mathbf{w} \in W\left(\left[t_{a}, t_{b}\right]\right)\right\} .
$$

We define the ISO occupancy as:

$\Gamma_{I S O}\left(\left[t_{i}, t_{j}\right]\right) \supseteq\left\{\mathbf{z} \mid \mathbf{x} \in \Gamma_{r}\left(t_{0}\right),\|\mathbf{z}-\mathbf{x}\| \leq\left(t_{b}-t_{0}\right) \cdot v_{h}^{I S O}\right\}$, where we take $v_{h}^{I S O}=1.6 \frac{\mathrm{m}}{\mathrm{s}}$, the maximum upper body speed used for the positioning of safeguards in [11].

$\Gamma_{r}$ captures the human model of all possible movements whereas $\Gamma_{I S O}$ describes the human movement expected according to the ISO standard. They are represented as capsules, which are the Minkowski sum $(\oplus)$ of a line segment $G$ and a sphere $H$, i.e. $G \oplus H=\{g+h \mid g \in G, h \in H\}$. The defining points of the capsule are the endpoints of $G$. Examples of capsules are shown in Fig. 5.

We can now formally define the safety criteria our trajectory guarantees to fulfil. The strong guarantee states:

Definition 2: STATIONARY CRITERION: Let $\mathbf{q}(t)$ be the joint positions of a robot at time $t$, and $\hat{F}(\mathbf{q}(t)) \subset \mathbb{R}^{3}$ be the set of Cartesian space it occupies. The stationary criterion is fulfilled if, for any nearby humans, the following holds:

$\forall t:\left(\hat{F}(\mathbf{q}(t)) \cap \Gamma_{I S O}(t)=\emptyset\right) \vee(\dot{\mathbf{q}}(t)=\mathbf{0})$.

Either the robot is already stationary or it can stop before the human could possibly come into contact with it. The weaker guarantee states:

Definition 3: REDUCED-SPEED CRITERION: Let $\mathbf{q}(t)$ be the joint positions of a robot at time $t$, and $\hat{F}(\mathbf{q}(t)) \subset$ $\mathbb{R}^{3}$ be the set of Cartesian space it occupies. Further, let $v(\mathbf{q}(t), \dot{\mathbf{q}}(t))$ be the maximum speed of any point on the robot in Cartesian space. The reduced-speed criterion is fulfilled if, for any nearby humans, the following holds:

$\forall t:\left(\hat{F}(\mathbf{q}(t)) \cap \Gamma_{r}(t)=\emptyset\right) \vee\left(v(\mathbf{q}(t), \dot{\mathbf{q}}(t)) \leq v_{\max }\right)$,

where $v_{\max }$ is a velocity limit to be specified in advance. Note that in contrast to Def. 2, we use $\Gamma_{r}$ and not $\Gamma_{I S O}$, since we account for all human motion.

We specify some conventions which we will use for the rest of the paper: $t_{k}$ is the current time and the short term plan to be verified runs from time $t_{k+1}$ to time $t_{e}$. The latter is the time the robot comes to a stop, i.e., the time after which the robot is safe according to the stationary criterion in Def. 2. Time $t_{e, v_{\max }}$ is the time on the short-term plan when the velocity of all points on the robot falls (and stays) below $v_{\max }$, i.e., from this time on, the robot is safe according to the reduced-speed criterion in Def. 3. We next present the methodology for our approach.

\section{TRAJECTORY MODIFICATION}

We describe how to modify the trajectory to guarantee the criteria in Defs. 2 and 3 by scaling the velocity. Algorithm 1 shows a high-level overview; Subsection III-A explains the planning of the short-term plan and Subsection III-B discusses the failsafe manoeuvre for the reduced-speed criterion. The algorithm is running continuously while the robot executes a long-term trajectory. In the following discussion, we show how to generate the short-term plan.

\section{A. Generation of short-term plan}

We here explain lines 4 and 5 of Algorithm 1. We express the global trajectory as a mapping of a time parameter $s$ to joint positions, $\boldsymbol{\xi}:\left[0, s_{f}\right] \rightarrow \mathcal{Q}$ where $\mathcal{Q} \subseteq \mathbb{R}^{n}$ is the joint space and $s_{f}$ is the final time parameter value of the longterm plan. Since the focus of this work is not path planning, 
the spatial paths of the robot are pre-programmed, and the robot executes a failsafe manoeuvre simply by scaling the rate of change of the time parameter $s$ to zero, while keeping accelerations and jerks within limits. In other words, at the end of the failsafe manoeuvre, $\dot{s}=0$. Time parameter scaling is a well-known way of temporally modifying trajectories, see e.g. [14].

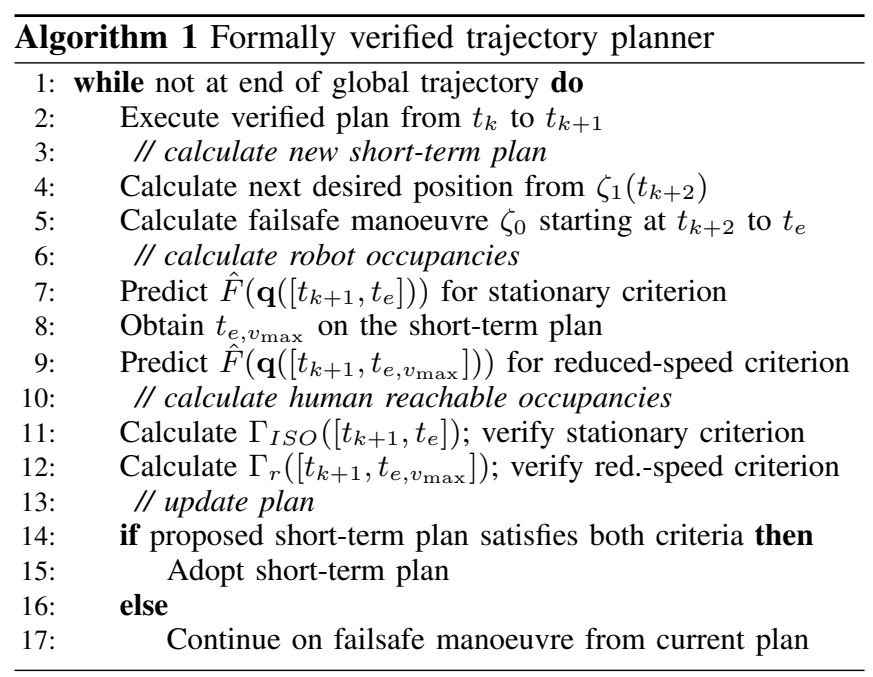

If the robot has previously been verified unsafe and has had to execute the failsafe manoeuvre, during time $t_{k+1}$ to $t_{k+2}$ it should also try to recover to full speed. It does this by planning a recovery manoeuvre, at the end of which $\dot{s}=1$. This is illustrated in Fig. 2. We next define a time-scaling plan, and then the failsafe and recovery manoeuvres:

Definition 4: TIME-SCALING PLAN: Given $s_{0}, \dot{s}_{0}$ and $\ddot{s}_{0}$ as the values of $s, \dot{s}$ and $\ddot{s}$ at time $t_{0}$, and $\eta \in\{0,1\}$, a timescaling plan starting at $t_{0}$ until $t_{1}$ is a monotone function $\zeta_{\eta}:\left[t_{0}, t_{1}\right] \rightarrow\left[0, s_{f}\right]$ where $\zeta_{\eta}\left(t_{0}\right)=s_{0}, \dot{\zeta}_{\eta}\left(t_{0}\right)=\dot{s}_{0}$ and $\ddot{\zeta}_{\eta}\left(t_{0}\right)=\ddot{s}_{0}, \dot{\zeta}_{\eta}\left(t_{1}\right)=\eta$ and $\ddot{\zeta}_{\eta}\left(t_{1}\right)=0$.

Definition 5: FAILSAFE AND RECOVERY MANOEUVRE: A failsafe manoeuvre $\zeta_{0}$ starting at $t_{0}$ is a time scaling plan where $\eta=0$. A recovery manoeuvre $\zeta_{1}$ starting at $t_{0}$ is a time scaling plan where $\eta=1$.

The short-term plan $\zeta:\left[t_{k+1}, t_{e}\right] \rightarrow\left[0, s_{f}\right]$ is planned thus: If from time $t_{k}$ to $t_{k+1}$ the robot is executing the failsafe manoeuvre, then we plan a recovery manoeuvre $\zeta_{1}$ starting at $t_{k+1}$. If the robot is already performing a previously-planned recovery manoeuvre from $t_{k}$ to $t_{k+1}$, we do not plan a new one. The position of the robot at time $t_{k+2}$ is therefore $\boldsymbol{\xi}\left(\zeta_{1}\left(t_{k+2}\right)\right)$. We then calculate a failsafe manoeuvre $\zeta_{0}$ starting at $t_{k+2}$ until $t_{e}$; the position of the robot at any time $t_{k+2}<t \leq t_{e}$ is $\boldsymbol{\xi}\left(\zeta_{0}(t)\right)$. This constitutes the short-term plan, which is shown in bold in Fig. 2 .

We next show how to generate the failsafe and the recovery manoeuvres $\zeta_{0}$ and $\zeta_{1}$, subject to joint acceleration and jerk limits. The Euclidean norm is denoted $\|\mathbf{z}\|$, and $|\mathbf{z}|$ is a vector of the absolute values of the elements of $\mathbf{z}$.

1) Planning manoeuvres with limited acceleration and jerk: We adapt the method from [15]. This finds a timeoptimal trajectory of joint values $\mathbf{q}$ in time, subject to limits

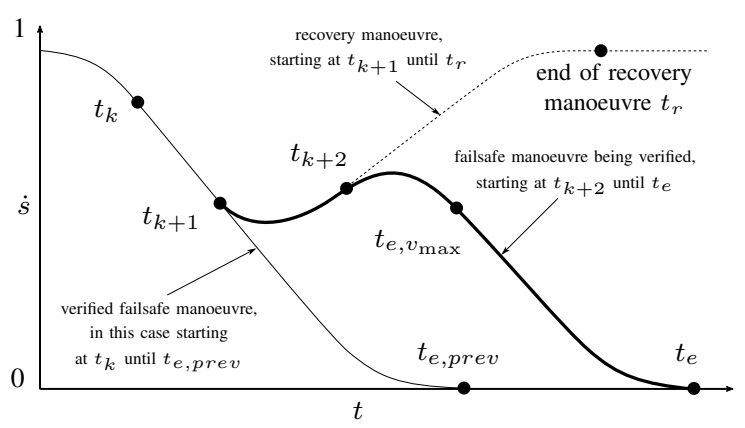

Fig. 2. Illustration of the planning of failsafe and recovery manoeuvres. From time $t_{k}$ to $t_{k+1}$ the robot executes the verified short term plan (here the failsafe manoeuvre) and plans the next short term plan (bold line), which consists of one step along the recovery manoeuvre until $t_{k+2}$ followed by a (new) failsafe manoeuvre.

on $\ddot{\mathbf{q}}$ and $\dddot{\mathbf{q}}$, and given the values of $\mathbf{q}, \dot{\mathbf{q}}$ and $\ddot{\mathbf{q}}$ at the start and $\dot{\mathbf{q}}$ and $\ddot{\mathbf{q}}$ at the end of the trajectory. Since the failsafe and recovery manoeuvres are not trajectories of joint positions but instead trajectories of $s$ in time, we use the same method to find a trajectory of $s$, subject to limits on $\ddot{s}$ and $\dddot{s}$. By setting these limits conservatively, we can account for maximum allowable joint accelerations and jerks.

We call $s_{0}$ the position at the start of the manoeuvre and $s_{1}$ the position at the end. The joint accelerations and jerks are $\ddot{\boldsymbol{\xi}}(s)$ and $\dddot{\boldsymbol{\xi}}(s)$, which we must guarantee to be less than some limits $\mathbf{a}_{m}$ and $\mathbf{j}_{m}$. Note that $\boldsymbol{\xi}, \mathbf{a}_{m}, \mathbf{j}_{m} \in \mathbb{R}^{n}$.

Theorem 1: Let $\boldsymbol{\theta}$ and $\boldsymbol{\lambda}$ be vectors, the elements of which are the maximum magnitude of $\frac{\mathrm{d}^{2} \xi}{\mathrm{d} s^{2}}$ and $\frac{\mathrm{d}^{3} \xi}{\mathrm{d} s^{3}}$ over the desired trajectory for each joint $i$, i.e., where $\theta_{i}, \lambda_{i}$ and $\xi_{i}$ are the $i^{t h}$ element of $\boldsymbol{\theta}, \boldsymbol{\lambda}$ and $\boldsymbol{\xi}$, then $\theta_{i}=\max _{s \in\left[0, s_{f}\right]}\left(\left|\frac{\mathrm{d}^{2} \xi_{i}}{\mathrm{~d} s^{2}}\right|\right)$ and $\lambda_{i}=\max _{s \in\left[0, s_{f}\right]}\left(\left|\frac{\mathrm{d}^{3} \xi_{i}}{\mathrm{~d} s^{3}}\right|\right)$. One can guarantee that $\ddot{\boldsymbol{\xi}}(s) \leq \mathbf{a}_{m}$ by setting $\ddot{s}_{m}$, the limit of $\ddot{s}$ over the manoeuvre, to:

$$
\ddot{s}_{m}=\max (\min (\mathbf{c}), 0), \quad \mathbf{c}=\frac{\mathbf{a}_{m}-\boldsymbol{\theta}}{\left|\frac{\mathrm{d} \boldsymbol{\xi}}{\mathrm{d} s}\right|_{s_{0}} \mid+\boldsymbol{\theta}\left(s_{1}-s_{0}\right)},
$$

where, for the above and following equation, division is elementwise, and $\min (\mathbf{z})$ returns the minimum element of the vector $\mathbf{z}$. Furthermore, one can guarantee $\dddot{\boldsymbol{\xi}}(s) \leq \mathbf{j}_{m}$ by setting $\dddot{s}_{m}$, the limit of $\dddot{s}$ over the manoeuvre, to:

$$
\dddot{s}_{m}=\max (\min (\mathbf{d}), 0), \quad \mathbf{d}=\frac{\mathbf{j}_{m}-\boldsymbol{\lambda}-3 \boldsymbol{\theta} \ddot{s}_{m}}{\left|\frac{\mathrm{d} \boldsymbol{\xi}}{\mathrm{d} s}\right|_{s_{0}} \mid+\boldsymbol{\theta}\left(s_{1}-s_{0}\right)} .
$$

Proof: Using the chain rule, we obtain the inequalities that must be satisfied by our choice of $\ddot{s}$ and $\dddot{s}$ :

$$
\begin{aligned}
& \ddot{\boldsymbol{\xi}}(s)=\frac{\mathrm{d}^{2} \boldsymbol{\xi}}{\mathrm{d} s^{2}} \dot{s}^{2}+\frac{\mathrm{d} \boldsymbol{\xi}}{\mathrm{d} s} \ddot{s} \leq \mathbf{a}_{m} ; \\
& \dddot{\boldsymbol{\xi}}(s)=\frac{\mathrm{d}^{3} \boldsymbol{\xi}}{\mathrm{d} s^{3}} \dot{s}^{3}+3 \frac{\mathrm{d}^{2} \boldsymbol{\xi}}{\mathrm{d} s^{2}} \dddot{s} \dot{s}+\frac{\mathrm{d} \boldsymbol{\xi}}{\mathrm{d} s} \dddot{s} \leq \mathbf{j}_{m} .
\end{aligned}
$$

Note that the following are stricter criteria than (1a) and (1b):

$$
\begin{aligned}
& \left|\frac{\mathrm{d}^{2} \boldsymbol{\xi}}{\mathrm{d} s^{2}} \dot{s}^{2}\right|+\left|\frac{\mathrm{d} \boldsymbol{\xi}}{\mathrm{d} s} \ddot{s}\right| \leq \mathbf{a}_{m} ; \\
& \left|\frac{\mathrm{d}^{3} \boldsymbol{\xi}}{\mathrm{d} s^{3}} \dot{s}^{3}\right|+\left|3 \frac{\mathrm{d}^{2} \boldsymbol{\xi}}{\mathrm{d} s^{2}} \ddot{s} \dot{s}\right|+\left|\frac{\mathrm{d} \boldsymbol{\xi}}{\mathrm{d} s} \dddot{s}\right| \leq \mathbf{j}_{m} .
\end{aligned}
$$


We rearrange (2a) and (2b) to obtain the requirements:

$$
\begin{aligned}
& |\ddot{s} \cdot \mathbf{1}| \leq \frac{\mathbf{a}_{m}-\left|\frac{\mathrm{d}^{2} \boldsymbol{\xi}}{\mathrm{d} s^{2}} \dot{s}^{2}\right|}{\left|\frac{\mathrm{d} \boldsymbol{d}}{\mathrm{d} s}\right|} \\
& |\dddot{s} \cdot \mathbf{1}| \leq \frac{\mathbf{j}_{m}-\left|\frac{\mathrm{d}^{3} \boldsymbol{\xi}}{\mathrm{d} s^{3}} \dot{s}^{3}\right|-\left|3 \frac{\mathrm{d}^{2} \xi}{\mathrm{d} s^{2}} \ddot{s} \ddot{s}\right|}{\left|\frac{\mathrm{d} \boldsymbol{\xi}}{\mathrm{d} s}\right|} .
\end{aligned}
$$

$1 \in \mathbb{R}^{n}$ is a vector of ones. We wish the inequalities of (3a) and (3b) to hold for $s \in\left[s_{0}, s_{1}\right]$. Consider first (3a). Since the global trajectory is known in advance, we can calculate $\boldsymbol{\theta}$, the maximum of $\frac{\mathrm{d}^{2} \xi}{\mathrm{d} s^{2}}$ over the global trajectory, and substitute this into $(3 \mathrm{a})^{1}$. Since $\dot{s} \in[0,1]$, the numerator of the righthand side attains a minimum at $\mathbf{a}_{m}-\boldsymbol{\theta}$. The global trajectory $\boldsymbol{\xi}$ is at least twice differentiable in $s$, so in the denominator we can use the Lagrange Remainder Theorem, which states $\frac{\mathrm{d} \boldsymbol{\xi}}{\mathrm{d} s}=\left.\frac{\mathrm{d} \boldsymbol{\xi}}{\mathrm{d} s}\right|_{s_{0}}+\left.\frac{\mathrm{d}^{2} \boldsymbol{\xi}}{\mathrm{d} s^{2}}\right|_{s^{*}}\left(s^{*}-s_{0}\right)$ for some $s^{*} \in\left[s_{0}, s_{1}\right]$. This is upper bounded by $\left.\frac{\mathrm{d} \boldsymbol{\xi}}{\mathrm{d} s}\right|_{s_{0}}+\boldsymbol{\theta}\left(s_{1}-s_{0}\right)$. A lower bound of the right-hand side of (3a) is therefore:

$$
\frac{\mathbf{a}_{m}-\boldsymbol{\theta}}{\left|\frac{\mathrm{d} \boldsymbol{\xi}}{\mathrm{d} s}\right|_{s_{0}} \mid+\boldsymbol{\theta}\left(s_{1}-s_{0}\right)}=\mathbf{c},
$$

and we take $\ddot{s}_{m}=\max (\min (\mathbf{c}), 0)$. Consider now (3b). By additionally using $\boldsymbol{\lambda}$, the maximum over the global trajectory, and the result $\ddot{s}_{m}$, we lower-bound ${ }^{2}$ the numerator of the right-hand side with $\mathbf{j}_{m}-\boldsymbol{\lambda}-3 \boldsymbol{\theta} \ddot{s}_{m}$. The denominator is the same as (3a). The right-hand side of (3b) has a lower bound:

$$
\frac{\mathbf{j}_{m}-\boldsymbol{\lambda}-3 \boldsymbol{\theta} \ddot{s}_{m}}{\left|\frac{\mathrm{d} \boldsymbol{\xi}}{\mathrm{d} s}\right|_{s_{0}} \mid+\boldsymbol{\theta}\left(s_{1}-s_{0}\right)}=\mathbf{d}
$$

and we choose $\dddot{s}_{m}=\max (\min (\mathbf{d}), 0)$.

As we do not know $s_{1}$ a priori, we use a conservative estimate which we expect to be longer than the actual end of the manoeuvre $s_{e}$; if it happens that $s_{e}>s_{1}$, we recalculate with an even more conservative estimate of $s_{1}$, until $s_{1} \geq s_{e}$.

We can now plan a failsafe or recovery manoeuvre in $s$ subject to the constraints that, at the end of the manoeuvre $\ddot{s}$ should be 0 and $\dot{s}$ should be 0 (failsafe) or 1 (recovery), and subject to the maximum acceleration and jerk $|\ddot{s}| \leq \ddot{s}_{m}$ and $|\dddot{s}| \leq \dddot{s}_{m}$. A method to plan a time-optimal manoeuvre subject to an arbitrary initial state, desired final state and limitations on acceleration and jerk is presented extensively in [15], and we refer the reader to this paper for details.

Having planned a short-term plan, we obtain $t_{e}$ as the time at the end of the plan. This is also the time at which the robot is first stationary and hence safe according to the stationary criterion (Def. 2). It remains to calculate $t_{e, v_{\max }}$, the time after which all points on the robot are moving slower than $v_{\max }$ and the robot is considered safe according to the reduced-speed criterion (Def. 3).

\section{B. Obtaining $t_{e, v_{\max }}$}

This subsection describes how to calculate $t_{e, v_{\max }}$ in line 8 of Algorithm 1, which is needed to verify the safety of a

\footnotetext{
${ }^{1}$ this is feasible as long as $\boldsymbol{\theta}<<\mathbf{a}_{m}$, otherwise the numerator of (4) is too small, or even negative, and hence $\ddot{s}_{m}$ is too restricted, or even zero.

${ }^{2}$ again, only feasible if $\boldsymbol{\lambda}<<\mathbf{j}_{m}$ for the same reason as above.
}

short-term plan regarding the reduced-speed criterion. For brevity, we denote the time parameter at a time $t_{k}$ on the short-term plan, $\zeta\left(t_{k}\right)$, by $s_{k}$. This short-term plan starts at position $s_{k+1}$ and time $t_{k+1}$ while the robot is moving from a trajectory position $s_{k}$ to $s_{k+1}$ in the time interval $\left[t_{k}, t_{k+1}\right]$ (cf. Fig. 2). An intermediate result for the calculation of $t_{e, v_{\max }}$ is the corresponding time parameter $s_{e, v_{\max }}$. While the failsafe manoeuvre of the short-term plan (cf. III-A) is planned such that the robot stops as soon as possible, we do not assume that the velocity in Cartesian space of all points on the robot is strictly decreasing during the manoeuvre.

Previously we calculated $t_{e}$ and the respective position on the trajectory $s_{e}$ (which corresponds to the end position $s_{1}$ of the failsafe manoeuvre in III-A). Starting at $s_{e}$ we backtrack on the trajectory until we either find a point where the robot is faster than the speed limit or we reach the start of the short-term plan $s_{k+1}$. If we find a point where the speed limit is violated, the position in the previous backtracking iteration with velocity below the limit is the sought $s_{e, v_{\max }}$. All points of the trajectory afterwards comply with the safety criterion. If backtracking terminates in the start position of the short-term plan at $s_{k+1}$, the whole short-term plan fulfils the criterion, $s_{e, v_{\max }}$ is just $s_{k+1}$, and the reduced-speed criterion does not inhibit the movement of the robot at all. Algorithm 2 shows pseudocode for the described procedure.

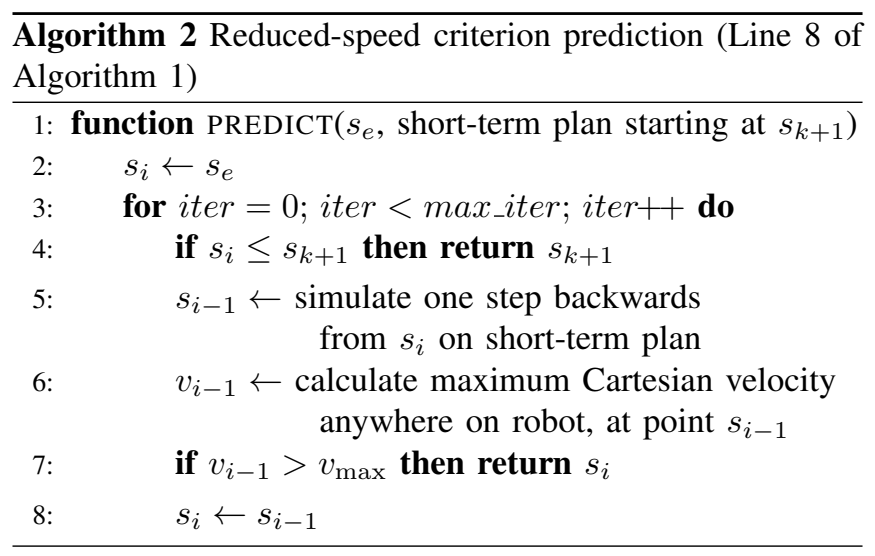

Although the ISO standard [10] only demands the TCP to be slower than $v_{\max }$, we present a novel, quick way to guarantee this for any point on the robot, as it is not certain where on the robot the human may collide with. Therefore we have to calculate the velocity of the fastest moving point on the robot to compare it to $v_{\max }$. We consider each robot segment as a capsule (cf. IV-A), calculate their maximum velocities separately and take the maximum of these.

To start off, we predict the movement of each capsule at the current position in the backtracking loop $s_{i-1}$ and represent it as a screw axis movement. This screw axis movement consists of the axis (some offset vector $\mathbf{o} \in \mathbb{R}^{3}$ and a normalized direction $\mathbf{n} \in \mathbb{R}^{3}$ ) as well as an angular velocity $\omega \in \mathbb{R}$ around the axis and a translational velocity $v \in \mathbb{R}$ along it. With the joint coordinates $\mathbf{q}=\boldsymbol{\xi}\left(s_{i-1}\right)$, the corresponding velocity $\dot{\mathbf{q}}=\dot{\boldsymbol{\xi}}\left(s_{i-1}\right)$ and the Jacobian matrix $J(\mathbf{q})$ we calculate the segment's movement at (without loss 


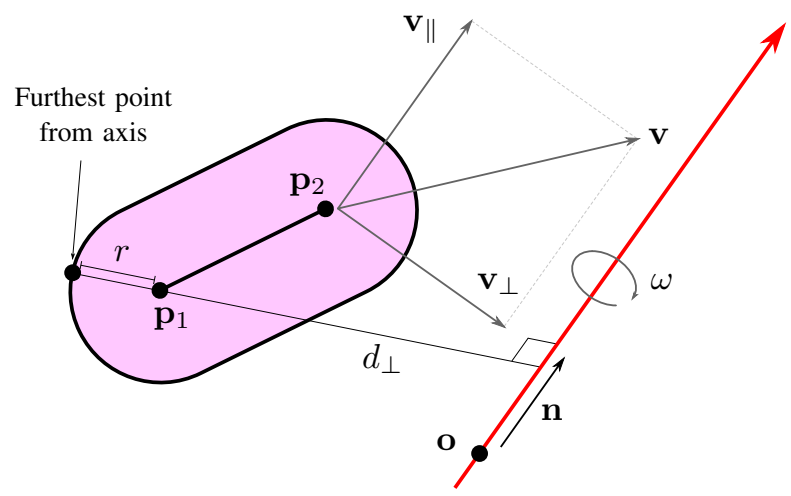

Fig. 3. Calculating the fastest moving point on the robot capsule. Shown in red is the screw axis. The fastest point is the furthest one from the axis. $\mathbf{p}_{1}$ and $\mathbf{p}_{2}$ are the defining points of the capsule. Other symbols are defined in the text.

of generality) $\mathbf{p}_{2}$ as twist $J(\mathbf{q}) \dot{\mathbf{q}}=\left(\begin{array}{l}\omega \\ v\end{array}\right)$. The twist consists of the angular velocity $\boldsymbol{\omega}=\omega \mathbf{n}$ (where $\|\mathbf{n}\|=1$ ) and a translational part $\mathbf{v} \in \mathbb{R}^{3}$. We decompose $\mathbf{v}$ into two parts, $\mathbf{v}_{\|}=\left(\mathbf{v}^{T} \cdot \mathbf{n}\right) \mathbf{n}$ parallel to $\mathbf{n}$ and $\mathbf{v}_{\perp}=\mathbf{v}-\mathbf{v}_{\|}$perpendicular to $\mathbf{n}$. The parallel part is the translational velocity of the screw axis $\mathbf{v}_{\|}=v \mathbf{n}$. The perpendicular part determines the offset $\mathbf{o}$ with the relation $\mathbf{v}_{\perp}=\boldsymbol{\omega} \times\left(\mathbf{p}_{2}-\mathbf{o}\right)$. This allows us to calculate $\mathbf{o}$ by considering the cross product as multiplication with a skew-symmetric matrix $\mathbf{v}_{\perp}=S(\boldsymbol{\omega})\left(\mathbf{p}_{2}-\mathbf{o}\right)$ and picking one of the solutions of the system of linear equations. A special case is a pure translation $(\omega=0)$, but in this case all points of the capsule move with the same speed, i.e. $v$.

Having calculated the screw axis movement we can now calculate the fastest moving point on the rigid body. The total velocity of a point $\mathbf{p}_{x}$ on the body is $\dot{\mathbf{p}}_{x}=v \mathbf{n}+\omega \mathbf{n} \times\left(\mathbf{p}_{x}-\right.$ $\mathbf{o}$ ), which also holds for the defining points of the capsule $\mathbf{p}_{1}$ and $\mathbf{p}_{2}$. The translational and angular terms of the sum are orthogonal, so the magnitude of $\dot{\mathbf{p}}_{x}$ can be calculated as:

$$
\left\|\dot{\mathbf{p}}_{x}\right\|=\sqrt{\|v \mathbf{n}\|^{2}+\left\|\omega \mathbf{n} \times\left(\mathbf{p}_{x}-\mathbf{o}\right)\right\|^{2}} .
$$

Finding the maximum of this for all points on a robot link comes down to finding the maximum of $\left\|\mathbf{n} \times\left(\mathbf{p}_{x}-\mathbf{o}\right)\right\|$, which is the perpendicular distance between the screw axis and the point $\mathbf{p}_{x}$.

The furthest point of the line segment $\overline{\mathbf{p}_{1} \mathbf{p}_{2}}$ to the screw axis is whichever of the defining points is further. Since we consider the rigid link to be a capsule, the furthest point on the capsule is a point on the surface of it, that is one capsule radius away from the further defining point. The maximum perpendicular distance is then:

$$
d_{\perp}:=\max \left(\left\|\mathbf{n} \times\left(\mathbf{p}_{1}-\mathbf{o}\right)\right\|,\left\|\mathbf{n} \times\left(\mathbf{p}_{2}-\mathbf{o}\right)\right\|\right)+r,
$$

where $r$ is the radius of the capsule. Fig. 3 illustrates the situation. By substituting (7) back into (6) the maximum velocity is finally:

$$
\max _{x}\left\|\dot{\mathbf{p}}_{x}\right\|=\sqrt{|v|^{2}+\left(|\omega| \cdot d_{\perp}\right)^{2}},
$$

which we can now compare against $v_{\max }$.

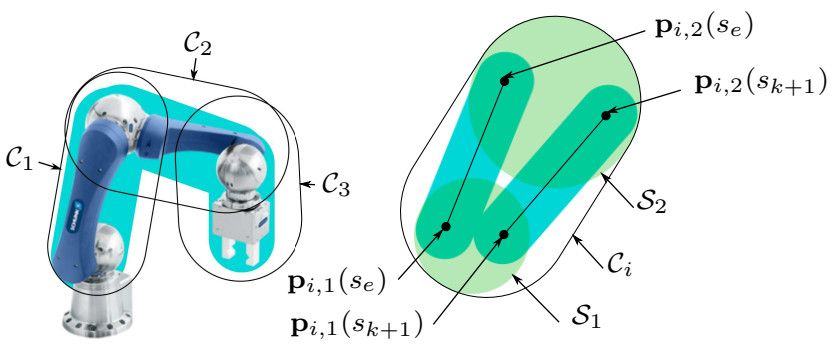

(a)

Fig. 4. (a) the enclosure of the occupancy of each link on the robot in capsules and (b) the generation of a capsule $\mathcal{C}_{i}$ to enclose the link occupancy during a short-term plan.

After obtaining $s_{e, v_{\max }}$, we find $t_{e, v_{\max }}=t_{e}-i t e r \cdot \Delta t$, where $\Delta t$ is the controller timestep. To ensure deterministic latencies, we limit the number of iterations to max_iter; if the limit is exceeded, the criterion is verified unsafe.

\section{PREDICTION OF OCCUPANCIES}

This section deals with obtaining the occupancies of the robot (Sec. IV-A) and the human (Sec. IV-B) during the short-term plan. Previous work on obtaining the occupancy of a kinematic chain in a conservative way [16] generates an overapproximative sphere-swept volume (SSV) which encloses the robot's occupancy over a section of a path. SSVs can be collision-checked by finding the minimum distance between them, e.g. using [17]. However, collision-checking of capsules is much faster and does not require iteration, e.g. as in [18]. Since the human reachable occupancy can also be obtained as capsules (see Sec. IV-B), we present a novel method to obtain the robot occupancy as capsules, in order to simplify and speed up collision-checking.

\section{A. Robot Occupancy}

In this subsection we describe how to obtain the occupancy of the robot, $\hat{F}\left(\mathbf{q}\left(\left[t_{k+1}, t_{e}\right]\right)\right)$ and $\hat{F}\left(\mathbf{q}\left(\left[t_{k+1}, t_{e, v_{\max }}\right]\right)\right)$, in lines 7 and 9 of algorithm 1 .

We first enclose each link of the robot in a capsule, as shown in Fig. 4a. The positions of the capsule end points are fixed on the robot and can be determined from forward kinematics. For each link $i$, we obtain a capsule $\mathcal{C}_{i}$ enclosing its occupancy over the short-term plan from $t_{k+1}$ to $t_{e}$, see Fig. 4b. We do this by calculating two balls $\mathcal{S}_{i, 1}$ and $\mathcal{S}_{i, 2}$ which enclose the spatial path of the defining points $\mathbf{p}_{i, 1}$ and $\mathbf{p}_{i, 2}$ of the link capsule from $t_{k+1}$ to $t_{e}$, enlarged by the radius of the link capsule. These balls are enclosed in a capsule $\mathcal{C}_{i}$. Since the defining points of the link capsule are in $\mathcal{C}_{i}$, by convexity, the entire link capsule is contained.

The endpoints of the link can be found from forward kinematics, i.e. $\mathbf{p}_{i, 1}$ and $\mathbf{p}_{i, 2}$ are functions of the joint positions, which in turn are a function of the path parameter $s$. For simplicity, we write: $\mathbf{p}_{i, 1}\left(s_{k+1}\right)$ and $\mathbf{p}_{i, 1}\left(s_{e}\right)$ for the position of $\mathbf{p}_{i, 1}$ at the path parameters at the start and end of the short-term plan; similarly for $\mathbf{p}_{i, 2}$.

Of course, the path traced by $\mathbf{p}_{i, 1}$ and $\mathbf{p}_{i, 2}$ during the short-term plan cannot be assumed to be a straight line. 
However, we approximate it by a straight line with some amount of deviation. Consider the point $\mathbf{p}_{i, 1}$, without loss of generality. In the below theorem and proof we adapt Proposition 1 in [19] to bound the maximum deviation from the line segment between $\mathbf{p}_{i, 1}\left(s_{k+1}\right)$ and $\mathbf{p}_{i, 1}\left(s_{e}\right)$, when the maximum value of $\left\|\frac{d^{2} \mathbf{p}_{i, 1}}{d s^{2}}\right\|$ is known. This value can be found from the given trajectory and is denoted by $\alpha_{i}$.

Theorem 2: Let $\mathbf{x}(\varsigma) \in \mathbb{R}^{3}$ be the position of a point at time parameter $\varsigma$ and suppose $\forall \varsigma:\|\ddot{\mathbf{x}}(\varsigma)\| \leq \alpha_{i}$. Let $\mathbf{x}_{0}$ and $\mathbf{x}_{f}$ be the known positions at time parameters $s=0$ and $s=s_{f}$ and $L$ be the line segment between them. For all $\varsigma \in\left[0, s_{f}\right], \mathbf{x}(\varsigma)$ is no further from $L$ than $\alpha_{i} \frac{s_{f}^{2}}{8}$.

Proof: From the equations of motion we have:

$$
\mathbf{x}(s)=\mathbf{x}_{0}+\dot{\mathbf{x}}(0) s+\int_{0}^{s} \int_{0}^{\varsigma^{\prime}} \ddot{\mathbf{x}}(\varsigma) d \varsigma d \varsigma^{\prime}
$$

Choosing $s=s^{*}$, we have the position of $\mathbf{x}\left(s^{*}\right)$ at an arbitrary time $s^{*} \in\left[0, s_{f}\right]$. The line segment $L$ can be expressed as the set $L=\left\{\mathbf{x}_{0}+\lambda\left(\mathbf{x}_{f}-\mathbf{x}_{0}\right) \mid \lambda \in[0,1]\right\}$. Substituting $s=s_{f}$ in (8), we obtain an expression for $\mathbf{x}_{f}=\mathbf{x}\left(s_{f}\right)$, and substituting this into the expression for the set $L$, and choosing $\lambda=\frac{s^{*}}{s_{f}}$, we see that the point $\mathbf{x}^{\prime}=\mathbf{x}_{0}+\dot{\mathbf{x}}(0) s^{*}+\frac{s^{*}}{s_{f}} \int_{0}^{s_{f}} \int_{0}^{\varsigma^{\prime}} \ddot{\mathbf{x}}(\varsigma) d \varsigma d \varsigma^{\prime}$ lies on $L$.

The difference $\mathbf{y}$ between $\mathbf{x}\left(s^{*}\right)$ and $\mathbf{x}^{\prime}$ is:

$$
\mathbf{y}=\frac{s^{*}}{s_{f}} \int_{0}^{s_{f}} \int_{0}^{\varsigma^{\prime}} \ddot{\mathbf{x}}(\varsigma) d \varsigma d \varsigma^{\prime}-\int_{0}^{s^{*}} \int_{0}^{\varsigma^{\prime}} \ddot{\mathbf{x}}(\varsigma) d \varsigma d \varsigma^{\prime}
$$

Observing that $\int_{0}^{s_{f}} \int_{0}^{\varsigma^{\prime}} \ddot{\mathbf{x}}(\varsigma) d \varsigma d \varsigma^{\prime}=\int_{0}^{s^{*}} \int_{0}^{\varsigma^{\prime}} \ddot{\mathbf{x}}(\varsigma) d \varsigma d \varsigma^{\prime}+$ $\int_{s^{*}}^{s_{f}} \int_{0}^{\varsigma^{\prime}} \ddot{\mathbf{x}}(\varsigma) d \varsigma d \varsigma^{\prime}$, we obtain:

$$
\mathbf{y}=\frac{s^{*}}{s_{f}} \underbrace{\int_{s^{*}}^{s_{f}} \int_{0}^{\varsigma^{\prime}} \ddot{\mathbf{x}}(\varsigma) d \varsigma d \varsigma^{\prime}}_{b}-\frac{s_{f}-s^{*}}{s_{f}} \underbrace{\int_{0}^{s^{*}} \int_{0}^{\varsigma^{\prime}} \ddot{\mathbf{x}}(\varsigma) d \varsigma d \varsigma^{\prime}}_{c}
$$

We observe that:

$$
\begin{aligned}
b & =\int_{s^{*}}^{s_{f}} \int_{0}^{s^{*}} \ddot{\mathbf{x}}(\varsigma) d \varsigma d \varsigma^{\prime}+\int_{s^{*}}^{s_{f}} \int_{s^{*}}^{\varsigma^{\prime}} \ddot{\mathbf{x}}(\varsigma) d \varsigma d \varsigma^{\prime} \\
& =\left(s_{f}-s^{*}\right) \int_{0}^{s^{*}} \ddot{\mathbf{x}}(\varsigma) d \varsigma+\int_{s^{*}}^{s_{f}} \int_{s^{*}}^{\varsigma^{\prime}} \ddot{\mathbf{x}}(\varsigma) d \varsigma d \varsigma^{\prime} ; \\
c & =\int_{0}^{s^{*}} \int_{0}^{s^{*}} \ddot{\mathbf{x}}(\varsigma) d \varsigma d \varsigma^{\prime}-\int_{0}^{s^{*}} \int_{\varsigma^{\prime}}^{s^{*}} \ddot{\mathbf{x}}(\varsigma) d \varsigma d \varsigma^{\prime} \\
& =s^{*} \int_{0}^{s^{*}} \ddot{\mathbf{x}}(\varsigma) d \varsigma-\int_{0}^{s^{*}} \int_{\varsigma^{\prime}}^{s^{*}} \ddot{\mathbf{x}}(\varsigma) d \varsigma d \varsigma^{\prime} ;
\end{aligned}
$$

since the inner integral of the first term of $b$ and $c$ do not depend on $\varsigma^{\prime}$ and can be taken outside the outer integral. Substituting (11) back into (10) we obtain:

$$
\mathbf{y}=\frac{s^{*}}{s_{f}} \underbrace{\int_{s^{*}}^{s_{f}} \int_{s^{*}}^{\varsigma^{\prime}} \ddot{\mathbf{x}}(\varsigma) d \varsigma d \varsigma^{\prime}}_{d}+\frac{s_{f}-s^{*}}{s_{f}} \underbrace{\int_{0}^{s^{*}} \int_{\varsigma^{\prime}}^{s^{*}} \ddot{\mathbf{x}}(\varsigma) d \varsigma d \varsigma^{\prime}}_{e}
$$

We observe that $d$ is the double-integral of acceleration from $\varsigma=s^{*}$ until $s_{f}$, which is upper-bounded by $\frac{\alpha_{i}\left(s_{f}-s^{*}\right)^{2}}{2}$. The expression $e$, which can be rewritten:

$\int_{0}^{s^{*}} \int_{\varsigma^{\prime}}^{s^{*}} \ddot{\mathbf{x}}(\varsigma) d \varsigma d \varsigma^{\prime}=\int_{0}^{s^{*}}\left(-\int_{s^{*}}^{\varsigma^{\prime}} \ddot{\mathbf{x}}(\varsigma) d \varsigma\right) d \varsigma^{\prime}=\int_{s^{*}}^{0} \int_{s^{*}}^{\varsigma^{\prime}} \ddot{\mathbf{x}}(\varsigma) d \varsigma d \varsigma^{\prime}$,

is the acceleration double-integrated "backward" from $\varsigma=s^{*}$ until 0 . This is upper-bounded by $\frac{\alpha_{i}\left(s^{*}\right)^{2}}{2}$. Both $s^{*}$ and $s_{f}-s^{*}$ are positive, so:

$$
\|\mathbf{y}\| \leq \frac{s^{*}}{s_{f}} \frac{\alpha_{i}\left(s_{f}-s^{*}\right)^{2}}{2}+\frac{s_{f}-s^{*}}{s_{f}} \frac{\alpha_{i}\left(s^{*}\right)^{2}}{2} .
$$

The right hand side attains a maximum of $\alpha_{i} \frac{s_{f}^{2}}{8}$ at $s^{*}=\frac{s_{f}}{2}$ (this can be seen by setting to zero the differential of the right hand side with respect to $\left.s^{*}\right)$. Hence the distance between $\mathbf{x}\left(s^{*}\right)$ and $L$ is no more than $\alpha_{i} \frac{s_{f}^{2}}{8}$.

We now show how to calculate $\mathcal{C}_{i}$. We denote the closed ball centered at $\mathbf{z}$ with radius $r$ as:

$$
B(\mathbf{z} ; r)=\{\mathbf{x} \mid\|\mathbf{x}-\mathbf{z}\| \leq r\} .
$$

The operators $\mathrm{CE}\left(b_{1}, b_{2}\right)$ and $\operatorname{BE}\left(b_{1}, b_{2}\right)$ standing for capsule enclosing and ball enclosing. These output the capsule or the ball enclosing the balls $b_{1}$ and $b_{2}$. Their detailed description is found in [13]. Formally, the occupancy of the link $i$ with radius $r_{l, i}$ is:

$$
\begin{aligned}
& r_{z, i}=\alpha_{i} \frac{\left(s_{e}-s_{k+1}\right)^{2}}{8}+r_{l, i} \\
& \mathcal{S}_{i, 1}=\operatorname{BE}\left(B\left(\mathbf{p}_{i, 1}\left(s_{k+1}\right) ; r_{z, i}\right), B\left(\mathbf{p}_{i, 1}\left(s_{e}\right) ; r_{z, i}\right)\right) \\
& \mathcal{S}_{i, 2}=\operatorname{BE}\left(B\left(\mathbf{p}_{i, 2}\left(s_{k+1}\right) ; r_{z, i}\right), B\left(\mathbf{p}_{i, 2}\left(s_{e}\right) ; r_{z, i}\right)\right) \\
& \mathcal{C}_{i}=\operatorname{CE}\left(\mathcal{S}_{i, 1}, \mathcal{S}_{i, 2}\right)
\end{aligned}
$$

By the property of convexity, since the link endpoints are enclosed, all points on the link $i$ are inside capsule $\mathcal{C}_{i}$.

\section{B. Prediction of Human Occupancy}

This subsection deals with the prediction of the human reachable occupancies $\Gamma_{r}$ and $\Gamma_{I S O}$ from lines 11 and 12 from Algorithm 1. The human arm is a nonlinear hybrid dynamical system, and its exact reachable set is impossible to calculate [20]. To still be able to formally verify the safety of the robot movement, one needs a tight, overapproximative prediction of human motion, meaning a prediction which includes all possible reachable states of the human, while excluding as many unreachable states as possible.

In [13] we present an approach to calculate 3 overapproximative predictions, which individually account for acceleration, velocity and position limits (since a prediction which would account for all limits simultaneously requires a hybrid model of human motion, and reachability analysis of hybrid systems is time consuming). Since each of these 3 predictions is a superset of the exact reachable occupancy of the human $\Gamma_{r}$, then if any of them is verified safe against the robot's short-term plan, then the exact reachable occupancy $\Gamma_{r}$ is also safe. We therefore perform verification on each of these occupancies and the reduced-speed criterion is verified, if any of these occupancies are verified safe. Due to space 


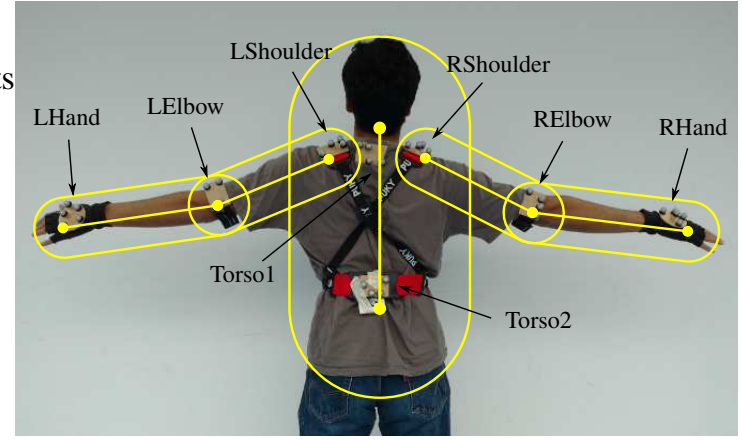

Fig. 5. Human reachable occupancy with the labelled marker clusters

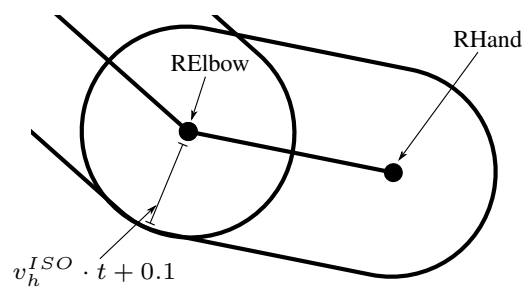

Fig. 6. Human reachable occupancy $\Gamma_{I S O}$

limitations, we do not detail the models here; the reader is referred to [13].

To calculate $\Gamma_{I S O}$, we assume a maximum speed of the human $\operatorname{arm} v_{h}^{I S O}=1.6 \frac{\mathrm{m}}{\mathrm{s}}$. We enclose the human in capsules, as shown in Fig. 5. These capsules' radii are specified such that they enclose all body parts-for the torso and head capsule, the radius is $0.3 \mathrm{~m}$ and for the upper arm and forearm capsules, the radii are both $0.1 \mathrm{~m}$. To calculate $\Gamma_{I S O}([0, t])$, we simply augment the radii by $v_{h}^{I S O} \cdot t$, shown in Fig. 6.

\section{EXPERIMENTS AND DISCUSSION}

We implemented and tested the described trajectory planner on a 6-DOF Schunk LWA 4P robot arm with a two-finger gripper mounted to the end effector. The robot is controlled by a Speedgoat SN2820 real-time target machine running a Simulink R2015b real-time kernel; robot and target machine communicate via CAN bus. Due to the Simulink kernel the robot control can be programmed in a Simulink model on a host computer connected to the target machine, and executed remotely. We use the robot arm in interpolated position mode $500 \mathrm{~Hz}$, i.e. a position command is sent every $2 \mathrm{~ms}$, and lowlevel control is performed by the motors in the joints.

\section{A. Human Tracking}

To track the upper body of the human we use six Vicon Vero 1.3 motion capture cameras that track reflecting markers at $100 \mathrm{~Hz}$. The camera data are aggregated by the Vicon Tracker 3.3 software, which tracks the position and orientation of coordinate systems attached to rigid clusters of markers. We made eight rigid marker clusters, each uniquely identifiable by Tracker 3.3, and affixed them to the body as shown in Fig. 5. A separate program using the Vicon DataStream SDK extracts the positions of the marker groups
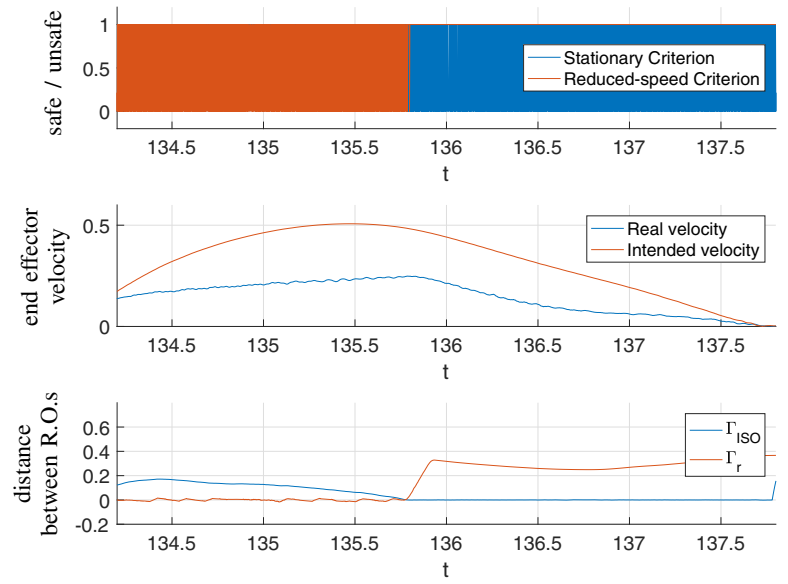

Fig. 7. Experimental results. Upper graph shows whether short-term plan could be verified safe; middle graph compares the real, reduced instantaneous end-effector velocity with the intended one, and the bottom graph shows the minimum distance between the reachable occupancies of human and robot at $t+t_{e}\left(\Gamma_{I S O}\right)$ and $t+t_{e, v_{\max }}\left(\Gamma_{r}\right)$.

relative to the robot's base from Tracker 3.3 and sends them to the target machine via UDP while it is executing the robot's routine. Although Tracker 3.3 cannot run on a realtime computer, the latency claimed by the manufacturers is very low at around $2.8 \mathrm{~ms}^{3}$. We took the latency from camera to real-time target machine as $5 \mathrm{~ms}$.

\section{B. Comparison}

In our tests the robot executed a predefined routine that simulated a pick-and-place task. The human then entered the robot's workspace and performed various movements with varying velocity to trigger the failsafe behaviour of the trajectory planner. The setup is shown in Fig. 8. To test the effectiveness and correctness of our approach we performed this trial multiple times with different values for $v_{\max }$ and compared it with an approach from previous work.

In this previous approach [6] our trajectory planner only used the stationary criterion to guarantee safety. We tested the approach from [6] separately with $\Gamma_{I S O}$ and $\Gamma_{r}$ to demonstrate its shortcomings compared to the approach presented in this paper. When using $\Gamma_{r}$ with the stationary criterion, the robot proved rather difficult to work with due to the high possible velocity and acceleration of the human. The human had to stand 2 to 3 meters away not to inhibit the robot. This can be seen in the video ${ }^{4}$ at 1:45. On the other hand with $\Gamma_{I S O}$ the trajectory planner provided mostly sufficient safety without being too restrictive, as long as the human moved slower than $v_{h}^{I S O}=1.6 \frac{\mathrm{m}}{\mathrm{s}}$. But as soon as the human moved faster than that, situations could occur where it is not entirely obvious whether the robot truly stopped completely before the human was able to reach it. This is again visible in the video ${ }^{4}$ at second 1:21. Finally, we tested the trajectory

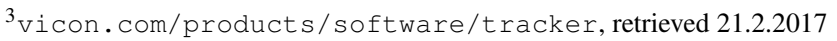

${ }_{4}$ ww6. in.tum.de/pub/Main/Pereira/CDC.mp 4
} 


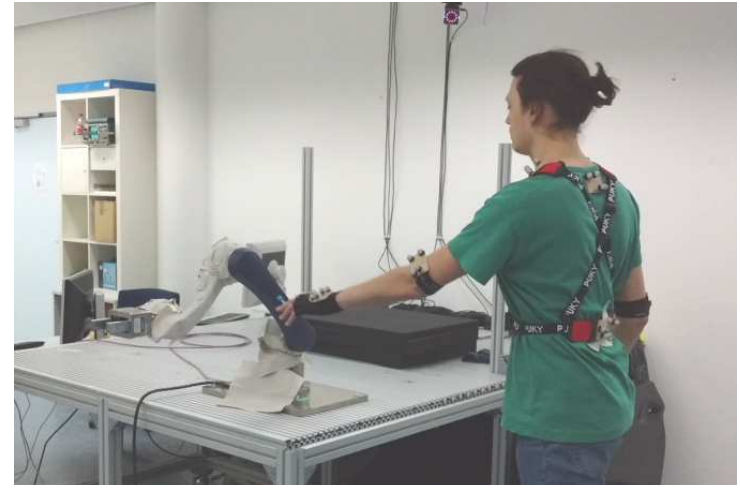

Fig. 8. Picture of the experimental trial.

planner with both criteria at the same time, once with the intended $v_{\max }=0.25 \frac{\mathrm{m}}{\mathrm{s}}$ and again with $v_{\max }=0.1 \frac{\mathrm{m}}{\mathrm{s}}$. With this the robot mostly behaved according to the stationary criterion, but in cases where it was faster than $v_{\max }$ and the human was standing respectively close had to slow down to abide to the reduced-speed criterion.

Fig. 7 shows a short section of the data recording of a test with $v_{\max }=0.1 \frac{\mathrm{m}}{\mathrm{s}}$. The upper graph shows the Boolean values of whether the short-term plans fulfil the two criteria, blue for the stationary criterion and orange for the reduced-speed criterion. They jump quickly between safe (1) and unsafe (0) as this slows the robot down just enough to separate the reachable occupancies. Note that this does not lead to chattering, since switching between failsafe and recovery manoeuvre changes only the jerks, which are limited to an acceptable value; joint accelerations stay continuous and joint velocities are differentiable. The middle graph shows the real velocity of the robot's end effector during the test and the intended velocity the robot should have had at that point. From around 134.2 seconds on, the robot must maintain a speed below the desired in order to respect the reduced-speed criterion. In case the human would have come any closer, the robot could have easily decelerated below $0.1 \frac{\mathrm{m}}{\mathrm{s}}$. At around 135.8 seconds it is the stationary criterion that limits the robot's speed. The bottom graph shows the minimum distances between the reachable occupancies during verification. Between 134.2 and 135.8 seconds, the distance between $\Gamma_{r}$ and the robot occupancy is around zero, meaning the reduced-speed criterion is often verified unsafe (top graph). At around time 135.8, the distance between $\Gamma_{I S O}$ and the robot occupancy goes down to zero, meaning the stationary criterion is often verified unsafe.

\section{CONCLUSIONS}

In this paper we present a method to formally plan and verify a safe robot trajectory online, without unnecessarily restricting robot motion. We guarantee both that, if the human moves as assumed by ISO standards, the robot can stop completely before impact, and that if not, the robot velocity will be under a pre-defined value at impact.

We test our approach in an experimental setup. We show that guaranteeing the robot can stop before impact while accounting for all human motion hinders robot operation, whereas only accounting for human movement assumed in ISO standards does not guarantee safety when the human moves faster than the assumptions. Our novel, dual-criterion online formal verification allows the robot to move efficiently while still guaranteeing safety, paving the way for formally safe robots which are also efficient workers.

\section{ACKNOWLEDGMENT}

The authors gratefully acknowledge financial support by the EC project UnCoVerCPS under grant number 643921.

\section{REFERENCES}

[1] H. Ding, K. Wijaya, G. Reißig, and O. Stursberg, "Online computation of safety-relevant regions for human robot interaction," in Proc. 43rd Intl. Symp. Robotics (ISR), 2012.

[2] J. Mainprice and D. Berenson, "Human-robot collaborative manipulation planning using early prediction of human motion," in Proc. IEEE/RSJ Int. Conf. Intell. Robots and Systems, 2013, pp. 299-306.

[3] H. Koppula and A. Saxena, "Anticipating human activities using object affordances for reactive robotic response," IEEE Trans. Pattern Anal. Mach. Intell., vol. 38, no. 1, pp. 14-29, 2015.

[4] S. Petti and T. Fraichard, "Safe motion planning in dynamic environments," in Proc. IEEE/RSJ Int. Conf. Intell. Robots and Systems, 2005, pp. 2210-2215.

[5] M. Althoff, "Reachability analysis and its application to the safety assessment of autonomous cars," Dissertation, TU München, 2010.

[6] A. Pereira and M. Althoff, "Safety control of robots under computed torque control using reachable sets," in Proc. IEEE Int. Conf. Robotics and Automation, 2015, pp. 331-338.

[7] A. Verl, A. Albu-Schäffer, O. Brock, and A. Raatz, Soft Robotics: Transferring Theory to Application. Springer, 2015.

[8] R. Rossi, M. Polverini, A. Zanchettin, and P. Rocco, "A pre-collision control strategy for human-robot interaction based on dissipated energy in potential inelastic impacts," in Proc. IEEE/RSJ Int. Conf. Intell. Robots and Systems, 2015, pp. 26-31.

[9] "Robots and robotic devices - collaborative robots," ISO/TS Standard 15066, International Organization for Standardization, 2016.

[10] "Robots and robotic devices - safety requirements for industrial robots - part 1: Robots," ISO Standard 10218-1, International Organization for Standardization, 2011.

[11] "Safety of machinery - positioning of safeguards with respect to the approach speeds of parts of the human body," ISO Standard 13855 International Organization for Standardization, 2010.

[12] A. Pereira and M. Althoff, "Overapproximative arm occupancy prediction for human-robot co-existence built from archetypal movements," in Proc. IEEE/RSJ Int. Conf. Intell. Robots and Systems, 2016.

[13] — - "Calculating human reachable occupancy for guaranteed collision-free planning," in Proc. IEEE/RSJ Int. Conf. Intell. Robots and Systems, 2017.

[14] D. Costantinescu and E. Croft, "Smooth and time-optimal trajectory planning for industrial manipulators along specified paths," Journal of robotic systems, vol. 17, no. 5, pp. 233-249, 2000.

[15] T. Kröger and F. Wahl, "Online trajectory generation: Basic concepts for instantaneous reactions to unforeseen events," IEEE Transactions on Robotics, vol. 26, no. 1, pp. 94-111, 2010.

[16] H. Täubig, B. Bäuml, and U. Frese, "Real-time swept volume and distance computation for self collision detection," in Proc. IEEE/RSJ Int. Conf. Intell. Robots and Systems, 2011, pp. 1585-1592.

[17] E. Gilbert, D. Johnson, and S. Keerthi, "A fast procedure for computing the distance between complex objects in three-dimensional space," IEEE J. Robotics and Automation, vol. 4, no. 2, pp. 193-203, 1988.

[18] C. Ericson, Real-Time Collision Detection. Morgan Kaufmann, 2004

[19] M. Zeestraten, A. Pereira, M. Althoff, and S. Calinon, "Online motion synthesis with minimal intervention control and formal safety guarantees," in Proc. IEEE Systems, Man and Cybernetics, 2016, pp. 2116-2121.

[20] A. Platzer and E. Clarke, "The image computation problem in hybrid systems model checking," in Hybrid Systems: Computation and Control, A. Bemporad, A. Bicchi, and G. Buttazzo, Eds. Springer, 2007, pp. $473-486$. 